\title{
Analytische Psychologie
}

Verena Rosetti-Gsell

\section{Spielen - Sprache der kindlichen Seele. Erkenne dein Kind im Spiel}

Freiburg i.B., Herder 1998

ISBN 3-451-26227-4, 158 S.; DEM 28.-

Wie der Untertitel nahe legt, ist das Buch für Eltern gedacht, die mehr über ihre Kinder wissen und sie auf dem schwierigen Weg des Heranwachsens begleiten wollen. Die Autorin ist jungianische Psychotherapeutin für Kinder und Jugendliche, Ergotherapeutin sowie Therapeutin für Psychomotorik. In dem Buch geht es nicht um vorgefertigte mehr oder weniger sinnvolle Spiele mit pädagogischem Wert, sondern um spontane, selbst entwickelte Spiele der Kinder, die aus deren eigenem Antrieb entstehen. Das Spiel ist die «Sprache der Kinder», indem es Wünsche, Hoffnungen, Ängste, Freude oder Mangel - also seelische Zustände und Entwicklungen - auf symbolische Weise direkt auszudrücken vermag. Für diese kreativen Ausdrucksweisen der Kinder möchte die Autorin die Eltern sensibel machen und ihnen bei deren Verständnis behilflich sein. Um die Dynamik zu erläutern, die im kindlichen Spiel Ausdruck findet, greift sie auf das Konzept der Transzendenten Funktion zurück. Diese ermöglicht die kreative Leistung der Psyche, wenn sie mit konflikthaften affektiven Bedürfnissen konfrontiert wird. Gerade im Spiel der Kinder wird die Wirkungsweise der Transzendenten Funktion so deutlich, indem die Kinder mit dem spontanen Spiel ihre alltäglichen Konflikte direkt darstellen und dabei versuchen, sie zu bewältigen. Die individuelle Darstellungsmöglichkeit der Kinder ist die eine Seite, die andere ist die Umgebung, in der das Spiel stattfindet und ganz besonders die fördernden oder hemmenden Einwirkungen der Bezugspersonen. Die Autorin beschreibt sehr schön und ohne moralischen Zeigefinger, wie Eltern über Verbot, verschiedene Formen von Bestrafung wie z.B. Liebesentzug oder Verständnis und Toleranz hemmenden oder fördernden Einfluss nehmen auf die Gestaltung im Spiel und somit auf den Umgang mit und die Verarbeitung von inneren Prozessen. Es ist ihr daran gelegen, Raum dafür zu schaffen oder zu erweitern, damit auch «schlimme oder böse Gedanken und Gefühle» Platz finden. Gerade bei als gefährlich erlebten Bedürfnissen, wie z.B. Eifersucht oder Beseitigungswünsche gegenüber Geschwisterrivalen, ist dies wichtig. Mit ihren Ausführungen möchte sie das Verstehen der Eltern fördern und deren Angst mildern, so dass die innere Realität ausgedrückt und geklärt werden kann und nicht durch erschreckte Reaktionen behindert wird. Denn erst ein akzeptierender und verstehender Umgang ermöglicht es, dass ängstigende Phantasien und Impulse auch integriert werden können und somit nicht verleugnet werden müssen. Dies sieht sie als Voraussetzung, die jeweils ursprüngliche Vitalität der Kinder zu erhalten, wobei sie nicht übersieht, dass auch den Erziehern Grenzen gesetzt sind, sich selbst nimmt sie dabei nicht aus.

Die einzelnen Kapitel tragen die Überschriften Verstecken, suchen, finden und fangen; Bewegungsspiele; Füllen, sammeln, horten, schenken, verkaufen und stehlen; Das Spiel mit den Elementen; Bauen und zerstören; mit Puppen spielen; Sich verwandeln und verkleiden;

\section{KARGER}

(C) 1996 S. Karger AG, Basel

Fax +41613061234

E-Mail karger@karger.ch

www. karger.com Accessible online at:

http://BioMedNet.com/karger 
Kämpfen, siegen; Waffen gebrauchen und Verkehrsspiel. Darin beschreibt die Autorin an Hand sehr anschaulicher Beispiele aus ihrer Praxis, eigener Erlebnisse und Erinnerungen aus dem privaten Bereich verschiedene Situationen und deren Verständnis auf dem Hintergrund von Ereignissen, Konflikten und lebensgeschichtlichen Zusammenhängen. An einzelnen Stellen greift sie auf theoretische Erläuterungen zurück und versteht es, die zitierten Autoren mit ausgewählten Aussagen immer in sehr verständlicher Weise einzubeziehen.

Für sehr wichtig halte ich auch die Kapitel, in denen sie auf die unterschiedlichen Spiele von Mädchen und Jungen eingeht. Bewaffnung, Angriff, Verteidigung, kämpferische Auseinandersetzung haben bei den Jungen die Funktion, männliche Selbstbestätigung und Identität zu gewinnen; vor allem dienen sie aber dazu, sich von der verschlingenden, primären Mütterlichkeit zu befreien. Erst später hilft der Kampf bei der ödipalen Auseinandersetzung. Sie betont die Wichtigkeit, auch den Mädchen die kämpferische Auseinandersetzung zuzugestehen, damit sich auch deren männliche Seiten lebendig und individuell entwickeln können.

Im Vordergrund ihrer Interpretation der kindllichen Spiele steht meiner Meinung nach jedoch zu häufig ein Aspekt, der sich auf das Bedürfnis nach Selbstentfaltung, Stärkung des Selbstwertgefühls, den Wünschen nach Geborgenheit der Kinder bezieht. Aggressive Impulse und Destruktivität finden zwar Erwähnung, kommen allerdings in der Darstellung ihrer Problematik zu kurz, was stellenweise den Eindruck erweckt, als sei die Therapeutin in ihren Therapien nicht auch mit schwierig zu handhabenden Gegenübertragungsgefühlen beschäftigt, als sei sie die immer nur verstehende und deshalb gute Mutter. Vielleicht ist dieser Kritikpunkt auch überzogen, da man nicht vergessen darf, dass dieses Buch in erster Linie für Eltern geschrieben und somit nicht der Ort ist, an den eine weitergehende theoretische Auseinandersetzung hingehört.

Toleranz gegenüber den kindlichen Spielen und deren Verstehen ist das Anliegen der Autorin, und es ist ihr auch gelungen, ihren Teil dazu beizutragen. Deshalb wünsche ich diesem sehr lesenswerten und sympathisch geschriebenen Buch, dass es unter Eltern, Erziehern, Lehrern und allen, die an Kindern interessiert sind, eine weite Verbreitung findet.

Ch. Braun-Demmer, Köln 